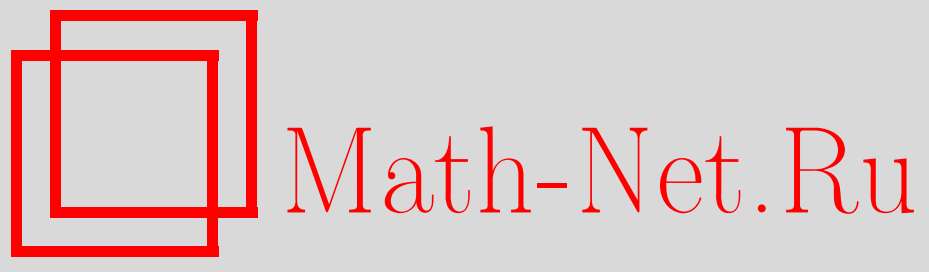

И. М. Щепочкина, Как реализовать алгебру Ли векторными полями, ТMФ, 2006, том 147, номер 3, 450-469

DOI: https://doi.org/10.4213/tmf1987

Использование Общероссийского математического портала Math-Net.Ru подразумевает, что вы прочитали и согласны с пользовательским соглашением http://www . mathnet.ru/rus/agreement

Параметры загрузки:

IP : 54.162 .127 .20

26 апреля 2023 г., 15:13:56 


\section{КАК РЕАЛИЗОВАТЬ АЛГЕБРУ ЛИ ВЕКТОРНЫМИ ПОЛЯМИ}

Описаны алгоритм вложения конечномерной алгебры (супералгебры) Ли в алгебру (супералгебру) Ли векторных полей, пригодный для основного поля любой характеристики, и способ выделения картановских, полных и частичных продолжений алгебр Ли векторных полей дифференциальными уравнениями. Алгоритм проиллюстрирован примером интерпретации Картана исключительной алгебры Ли $\mathfrak{g}(2)$ в виде алгебры Ли, сохраняющей некоторое неинтегрируемое распределение, а также некоторыми другими примерами.

Ключевые слова: картановское продолжение, неинтегрируемые распределения, $\mathfrak{g}(2)$ структура.

\section{1. ВВЕДЕНИЕ}

В статье приводится алгоритм, явно описывающий вложение произвольной абстрактной $\mathbb{Z}$-градуированной алгебры Ли (или супералгебры Ли) $\mathfrak{n}:=\bigoplus_{k \geqslant-d} \mathfrak{n}_{k}$ такой, что

$$
\mathfrak{n}_{-1} \quad \text { порождает } \quad \mathfrak{n}_{-}:=\bigoplus_{k<0} \mathfrak{n}_{k}, \quad \operatorname{dim} \mathfrak{n}_{-}<\infty
$$

в алгебру Ли (соответственно, супералгебру Ли) полиномиальных векторных полей над полем $\mathbb{K}$ любой характеристики $p^{1)}$. В качестве иллюстрации алгоритма рассмотрено вложение в векторные поля алгебры Ли $\mathfrak{g}(2)^{2)}$, не зависящее от характеристики поля.

По существу, мы возвращаемся к классическому картановскому [1] пониманию алгебры Ли как алгебры Ли векторных полей, сохраняющих некоторые геометрические структуры, в особенности, неинтегрируемые распределения.

В случае характеристики $p>0$ Грозман и Лейтес использовали настоящий алгоритм не только для получения новых реализаций известных, но плохо изученных простых алгебр Ли, но и для нахождения новых примеров [2]. В частности, важную

1)Хотя $p$ обозначает и характеристику поля, и четность, а иногда используется и в качестве индекса, из контекста всегда понятно, что имеется в виду.

2) Мы будем обозначать простую исключительную алгебру Ли аналогично простым алгебрам, образующим серии, как $\mathfrak{s l}(n)$; тем самым мы избежим путаницы между алгеброй $\mathfrak{g}(2)$ и компонентой $\mathfrak{g}_{2}$ градуировки два произвольной $\mathbb{Z}$-градуированной алгебры $\mathfrak{g}$.

*Независимый московский университет, Москва, Россия. E-mail: irina@mccme.ru 
роль в [2] играет приведенная в данной статье реализация алгебры Ли типа $\mathfrak{g}(2)$ векторными полями. В настоящее время алгоритм реализован в пакете Грозмана "SuperLie" [3].

Для полей характеристики $p=0$ полные продолжения в случае градуировки глубины $d>1$ обычно описываются как алгебры Ли, сохраняющие некоторое распределение. Поскольку предложенный алгоритм не зависит от характеристики поля, мы и для $p>0$ приводим явный вид распределения, сохраняемого полным продолжением. Тем самым, мы даем положительный и явный ответ на вопрос работы [4] о возможности выделения полных продолжений с помощью системы уравнений Пфаффа.

Важной особенностью ситуации с супералгебрами Ли и алгебрами Ли над полем характеристики $p>0$ является наличие нетривиальных частичных продолжений, приводящих к простым алгебрам, чего никогда не бывает в случае вещественных или комплексных алгебр Ли [5]. В настоящей статье показано, как явно описать такие продолжения с помощью дифференциальных уравнений. В работах [6], [7] этот способ использован для явного описания двух исключительных простых супералгебр Ли векторных полей. В настоящей статье приводятся уравнения, выделяющие еще одну простую исключительную супералгебру Ли, получающуюся как частичное продолжение.

Другие работы на данную тему. В работах [8], [9] явно вычислены геометрические структуры, сохраняемые некоторыми простыми исключительными супералгебрами Ли векторных полей, а также простыми исключительными алгебрами Ли.

Задача реализации алгебры Ли операторами рождения-уничтожения тесно связана с задачей, рассмотренной в настоящей статье. Последние результаты и обзор других работ на эту тему можно найти в [10], [11].

Постановка задачи, известные факты и некоторые соображения. Предположим, что $\mathfrak{n}:=\bigoplus_{k=-d}^{-1} \mathfrak{n}_{k}-$ это $n$-мерная $\mathbb{Z}$-градуированная алгебра (или супералгебра) Ли глубины $d>1$, удовлетворяющая условию (1). Пусть

$$
f: \mathfrak{n} \rightarrow \mathfrak{v e c t}(n)=\mathfrak{d e r} \mathbb{K}\left[x_{1}, \ldots, x_{n}\right]
$$

- вложение. Будем работать с полиномиальными векторными полями, т.е. локализовать ситуацию в окрестности начала координат. Обозначим через $f(\mathfrak{n})(0)$ пространство 0-струй в точке 0 векторных полей из образа $f(\mathfrak{n})$.

ЗАДАЧА 1. Вложить $\mathfrak{n}$ в $\mathfrak{v e c t}(n)$ так, чтобы $\operatorname{dim} f(\mathfrak{n})(0)=n=\operatorname{dim} \mathfrak{n}$.

Грубо говоря, мы хотим, чтобы образ алгебры $\mathfrak{n}$ действовал транзитивно (без инвариантных подмногообразий) в окрестности точки 0, или, на алгебраическом языке, чтобы по модулю векторных полей, обращающихся в 0 в начале координат, образ нашей алгебры содержал бы все частные производные $\partial_{x_{1}}, \ldots, \partial_{x_{n}}$. Такое вложение задает нестандартную ${ }^{3)}$ градуировку глубины $d$ алгебры Ли $\mathfrak{v e c t}(n)$. Мы будем обозначать алгебру Ли $\mathfrak{v e c t}(n)$, рассматриваемую в этой нестандартной градуировке, через $\mathfrak{v}=\bigoplus_{k=-d}^{\infty} \mathfrak{v}_{k}$. Пусть $\mathfrak{g}_{-}-$это образ алгебры $\mathfrak{n}$ в $\mathfrak{v}$, т.е. $\mathfrak{g}_{-} \subset \mathfrak{v}_{-}:=\bigoplus_{k<0} \mathfrak{v}_{k}$.

\footnotetext{
3) Градуировка $\operatorname{deg} x_{i}=1$ для всех $i$, ассоциированная с $(x)$-адической фильтрацией, называется стандартной; любая другая градуировка - нестандартной.
} 
ЗАДАчА 2. Вычислить полное алгебраическое продолжсене алгебры $\mathfrak{g}_{-}$, т.е. найти максимальную подалгебру $\left(\mathfrak{g}_{-}\right)_{*}=\bigoplus_{k \geqslant-d} \mathfrak{g}_{k} \subset \mathfrak{v}$ с заданной отрицательной частью.

ЗАДАчА 3. Выделить частичное продолжение алгебры $\mathfrak{g}_{-}$в $\left(\mathfrak{g}_{-}\right)_{*}$.

В частности, если задана не только алгебра $\mathfrak{n}$, но и некоторая подалгебра $\mathfrak{n}_{0} \subset$ $\mathfrak{d e r} \mathfrak{r}_{0} \mathfrak{n}$, где $\mathfrak{d e r}_{0} \mathfrak{n}-$ подалгебра Ли в $\mathfrak{d e r}(\mathfrak{n})$, состоящая из дифференцирований, сохраняющих $\mathbb{Z}$-градуировку, мы автоматически получаем вложение $\mathfrak{n}_{0} \subset \mathfrak{g}_{0}$, которое определяет следующие частные случаи задачи 3.

ЗАДАчА 3.1. Пусть вложение $\mathfrak{n}_{0} \subset \mathfrak{g}_{0}$ строгое. Выделить в $\left(\mathfrak{g}_{-}\right)_{*}$ подалгебру $\mathfrak{n}_{0}$ в $\mathfrak{g}_{0}$, а также ее обобщенное картановское продолжение $\left(\mathfrak{g}_{-}, \mathfrak{n}_{0}\right)_{*}$, другими словами, максимальную подалгебру в алгебре $\mathfrak{v}$ с данной неположителъной частью.

ЗАДАЧА 3.2. Пусть $\mathfrak{n}_{0}=\mathfrak{g}_{0}$, но компонента $\mathfrak{g}_{1}$ образует приводимый $\mathfrak{g}_{0}$-модуль с подмодулем $\mathfrak{h}_{1}$. Выделить максимальную подалгебру $\left(\mathfrak{g}_{-} \oplus \mathfrak{g}_{0} \oplus \mathfrak{h}_{1}\right)_{*} \subset \mathfrak{v}$ с данной "начальной частью" (компонентами градуировки $\leqslant 1$ ).

ЗАДАчА 3.3. В наибольшей общности, выделить в алгебре $\mathfrak{v}$ максимальную подалгебру $\mathfrak{h}_{*}=\bigoplus_{k \geqslant-d} \mathfrak{h}_{k}$ с данной начальной частью $\mathfrak{h}=\mathfrak{g}_{-} \oplus\left(\bigoplus_{0 \leqslant k \leqslant K} \mathfrak{h}_{k}\right)$.

Естественно, начальная часть $\mathfrak{h}$ должна быть согласована со скобкой: $\left[\mathfrak{h}_{i}, \mathfrak{h}_{j}\right] \subset$ $\mathfrak{h}_{i+j}$ для всех $i, j$ таких, что $i+j \leqslant K$. При $k>K$ компоненты $\mathfrak{h}_{k}$ определяются рекуррентно:

$$
\mathfrak{h}_{k}=\left\{X \in \mathfrak{g}_{k} \mid\left[X, \mathfrak{g}_{-1}\right] \subset \mathfrak{h}_{k-1}\right\} .
$$

Для этих компонент включение $\left[\mathfrak{h}_{k}, \mathfrak{h}_{l}\right] \subset \mathfrak{h}_{k+l}$ доказывается индукцией по сумме $k+l$ с учетом условия $(1)$, и это гарантирует нам, что сумма $\mathfrak{h}_{*}:=\mathfrak{g}_{-} \oplus\left(\bigoplus_{0 \leqslant k} \mathfrak{h}_{k}\right)$ является подалгеброй в алгебре $\mathfrak{v}$. Алгебра Ли $\mathfrak{h}_{*}$ является обобщенным картановским продолжением.

ЗАмечАниЕ. Фактически для простых алгебр Ли над $\mathbb{R}$ или $\mathbb{C}$ число $K$ всегда не превосходит 1 , но для char $\mathbb{K}>0$ или для супералгебр Ли случай $K>1$ также возможен даже для простых алгебр.

Обсуждение. Если группа Ли $N$ с алгеброй Ли п задана явно, т.е. если мы знаем явные формулы для произведения элементов группы в некоторых координатах, то никакой проблемы с описанием вложения $\mathfrak{n} \subset \mathfrak{v e c t}(n)$ нет: алгебра Ли левоинвариантных (или правоинвариантных) векторных полей на группе $N$ изоморфна $\mathfrak{n}$. Это, по существу, одно из определений алгебры Ли группы $N$. Если группа $N$ не задана явно, то описание вложения $\mathfrak{n} \subset \mathfrak{v e c t}(n)$ является частью задачи восстановления группы Ли по ее алгебре Ли (в тех случаях, когда вообще имеет смысл говорить о группе Ли). Конечно, формула Кэмпбелла-Хаусдорфа помогает решить эту задачу. К сожалению, несмотря на свою важность в теоретических исследованиях формула Кэмпбелла-Хаусдорфа плохо приспособлена к явным вычислениям.

Для $\mathbb{K}=\mathbb{R}$ и $\mathbb{K}=\mathbb{C}$ еще один метод построения вложения $\mathfrak{n} \subset \mathfrak{v e c t}(n)$ и восстановления группы Ли по ее алгебре Ли состоит в интегрировании уравнений МаурераКартана [12]. Хотя алгоритм, который предлагается в данной работе, не использует группу Ли алгебры Ли $\mathfrak{n}$ и применим даже в тех случаях, когда о группе вообще 
говорить не приходится, именно понимание алгебры Ли как алгебры левоинвариантных векторных полей на группе дает нам ключевые соображения.

Другие алгоритмы вложения $\mathfrak{n} \subset \mathfrak{v e c t}(n)$, основанные на явном описании действия алгебры Ли n в универсальной обертывающей $U(\mathfrak{n})$, см. в работах [10], [11].

Перечислим теперь основные соображения, на которых основан алгоритм.

СООБРАЖЕНИЕ 1. Пусть $X_{1}, \ldots, X_{n}$ - векторные поля, линейно независимые в каждой точке $n$-мерной (супер)области, и

$$
\left[X_{i}, X_{j}\right]=\sum_{k} c_{i j}^{k} X_{k}, \quad c_{i j}^{k} \in \mathbb{K} .
$$

Пусть $\omega^{1}, \ldots, \omega^{n}$ - двойственный базис дифференциальных 1-форм, т.е. $\omega^{i}\left(X_{j}\right)=$ $\delta_{j}^{i}$. Тогда (и это стандартное упражнение)

$$
d \omega^{k}=-\frac{1}{2} \sum_{i, j} c_{i j}^{k} \omega^{i} \wedge \omega^{j}=-\sum_{i<j} c_{i j}^{k} \omega^{i} \wedge \omega^{j}
$$

и наоборот, если 1-формы $\omega^{1}, \ldots, \omega^{n}$ удовлетворяют соотношению (4), двойственные к ним векторные поля $X_{1}, \ldots, X_{n}$ удовлетворяют соотношению (3).

Заметим, что хотя в суперслучае формула

$$
d \omega(X, Y)=X \omega(Y)-Y \omega(X)-\omega([X, Y])
$$

требует коррекции с помощью изменения знаков, соотношения (4) остаются справедливыми и для супералгебр: дополнительные знаки в соотношении (5), появляющиеся в силу суперприроды его компонент, не меняют вид формулы (4).

Напомним, что если векторные поля $X_{i}$ образуют базис левоинвариантных векторных полей группы $N$, то соотношения (4) называются уравнениями МаурераКартана; в этом случае $c_{i j}^{k}$ - структурные константы алгебры Ли $\mathfrak{n}$.

Если $\omega^{i}=\sum_{k} V_{k}^{i}(x) d x^{k}$, то соотношения (4) переписываются в виде уравнений на функции $V_{k}^{i}$ :

$$
\partial_{j} V_{i}^{k}-\partial_{i} V_{j}^{k}=\sum_{p, q} c_{p q}^{k} V_{i}^{p} V_{j}^{q}
$$

СООБРАЖЕНИЕ 2. В вещественном и комплексном случаях уравнения (6) легко интегрируются в "хороших" координатах для любой алгебры Ли $\mathfrak{n}$ (не только нильпотентной). А именно введем функции

$$
W_{j}^{i}(t, x)=t V_{j}^{i}\left(e^{t x}\right), \quad t \in \mathbb{R}, \quad x \in \mathfrak{n}^{*} .
$$

Другими словами, мы должны интегрировать уравнения (6) вдоль однопараметрических подгрупп. Легко проверить (см. книгу [12]), что функции $W$ удовлетворяют обыкновенным дифференциальным уравнениям

$$
\frac{d W_{j}^{i}}{d t}=\delta_{j}^{i}+\sum_{p, q} c_{p q}^{i} W_{j}^{p} x^{q}
$$

с начальными условиями $W_{j}^{i}(0, x)=0$. 
На самом деле, в нильпотентном случае система (6) настолько проста, что нет нужды выбирать какие-то специальные координаты и вводить вспомогательные функции $W_{i}^{k}$. Ее можно проинтегрировать и непосредственно, причем для любого основного поля. Это непосредственное решение системы (6) позволяет нам построить вложение $\mathfrak{n} \rightarrow \mathfrak{v e c t}(n)$, наиболее пригодное для наших целей ${ }^{4)}$, а также найти все возможные вложения.

Выберем в алгебре Ли $\mathfrak{n}$ базис $B=\left\{e_{1}, \ldots, e_{n}\right\}$, согласованный с градуировкой. Это означает, что первые $n_{1}$ его элементов образуют базис компоненты $\mathfrak{n}_{-1}$, следующие $n_{2}$ элементов - базис компоненты $\mathfrak{n}_{-2}$ и т.д. Набор индексов, отвечающих базисным элементам компоненты $\mathfrak{n}_{-s}$ обозначим $I_{s}$. Пусть $I=\bigcup I_{s}, c_{i j}^{k}-$ структурные константы в выбранном базисе:

$$
\left[e_{i}, e_{j}\right]=\sum_{k} c_{i j}^{k} e_{k},
$$

а $x_{1}, \ldots, x_{n}-$ координаты, определяемые базисом $B$. Нестандартная $\mathbb{Z}$-градуировка $\mathfrak{v}=\bigoplus_{k \geqslant-d} \mathfrak{v}_{k}$ алгебры $\mathfrak{v e c t}(n)$, согласованная с $\mathbb{Z}$-градуировкой алгебры $\mathfrak{n}$, определяется следующим образом:

$$
\operatorname{deg} x_{i}=s, \quad i \in I_{s} .
$$

Пусть $X_{i} \in \mathfrak{v}$ есть образ элемента $e_{i}$ в этом вложении. Тогда значение поля $X_{i}$ в нуле равно $\partial_{x_{i}}:=\partial_{i}$, а значение двойственной формы $\omega^{i}$ в нуле равно $d x^{i}$. Если $i \in I_{s}$, то поле $X_{i}$ и форма $\omega^{i}$ однородны и имеют степени $-s$ и $s$, соответственно. Мы получаем

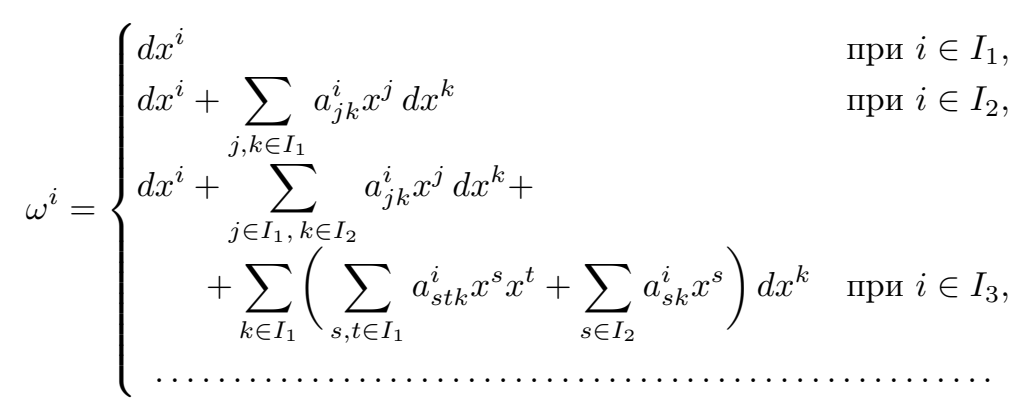

Наличие градуировки гарантирует автоматическое выполнение части уравнений (6). Так, при $k \in I_{1}$ все функции $V_{i}^{k}$ уже известны: $V_{i}^{k}=\delta_{i}^{k}$. Они удовлетворяют уравнениям (6), поскольку $c_{i j}^{k}=0$ для всех $k \in I_{1}$.

Для $k \in I_{2}$ правые части уравнений (6) содержат только функции $V_{i}^{p}, V_{j}^{q}$ с $p, q \in I_{1}$, т.е. уже известные функции. В результате получается система линейных уравнений на коэффициенты $a_{j k}^{i}$. Конечно, эта система является сильно неопределенной. Найдя все ее решения, мы найдем все возможные формы $\omega^{i}, i \in I_{2}$.

\footnotetext{
4) Например, непонятно, почему даже авторы работы [10] испытывали затруднения в применении каждого из трех предложенных ими алгоритмов. Автором данной работы были проверены все эти алгоритмы - они работают. Единственное объяснение, которое автор может привести, отвечая на вопросы, заданные ему Грозманом и Лейтесом, состоит в том, что формулы в [10] фиксированы, и некоторые из них содержат деления. А что делать, если вы работаете над полем конечной характеристики и хотите избежать деления (на 2 или 3) в коэффициентах?! Мы предлагаем пользователю возможность выбрать вложение по вкусу.
} 
Однако нам нужен лишь один набор форм, поэтому мы можем выбрать какое-то одно из решений. После того как функции $V_{i}^{k}$ для $k \in I_{1} \cup I_{2}$ найдены, их можно подставить в уравнения (6) на функции $V_{i}^{k} \mathrm{c} k \in I_{3}$ и т.д. В результате мы найдем все формы $\omega^{1}, \ldots, \omega^{n}$. Тождество Якоби гарантирует нам совместность системы.

СоОБРАЖЕНИЕ 3. Как въделять полные продолжения? В вещественном случае односвязная группа Ли $N$ с алгеброй Ли $\mathfrak{n}$, левоинвариантными формами $\omega^{i}, \quad i \in$ $I$, и структурными константами $c_{i j}^{k}$, определяемыми соотношениями (4), обладает следующим универсальным свойством.

Теорема [13]. Если $M$ - гладкое многообразие с набором линейно независимых в каждой точке дифберенциальных 1-форм $\alpha^{i}$, удовлетворяющих соотношениям (4) c теми же константами $c_{i j}^{k}$, то для любой точки $x \in M$ существуют такая окрестность $U$ и диффеоморфизм $f: U \rightarrow N$, что $\alpha^{i}=f^{*}\left(\omega^{i}\right)$. Два таких дифбеоморфизма отличаются левым сдвигом.

Следовательно, как только мы найдем формы $\omega^{i}$, удовлетворяющие соотношениям (4), мы можем считать их левоинвариантными формами на группе $N$, а дуальные векторные поля $X_{i}$ - левоинвариантными векторными полями.

Пусть $Y_{1}, \ldots, Y_{n}$ - правоинвариантные векторные поля такие, что $X_{i}(e)=Y_{i}(e)$, и $\theta^{1}, \ldots, \theta^{n}$ - двойственные к ним правоинвариантные 1-формы.

Ясно, что линейные оболочки обоих наборов полей $\left\{X_{i}\right\}$ и $\left\{Y_{i}\right\}$ являются изоморфными $\mathfrak{n}$ подалгебрами алгебры Ли $\mathfrak{v}_{-}$всех векторных полей на группе $N$ отрицательной нестандартной градуировки.

Определим на $N$ правоинвариантное распределение $\mathcal{D}$ такое, что $D(e)=\mathfrak{n}_{-1}$. Ясно, что $\mathcal{D}$ выделяется системой уравнений на поле $X \in \mathfrak{v e c t}(n)$ :

$$
\theta^{i}(X)=0, \quad i \in I_{2} \cup I_{3} \cup \cdots \cup I_{d} .
$$

Поскольку лево- и правоинвариантные векторные поля на группе Ли всегда коммутируют друг с другом, каждое поле $X_{j}$ сохраняет распределение $\mathcal{D}$, а значит, и вся алгебра Ли $\mathfrak{g}_{-}$сохраняет $\mathcal{D}$. Более того, из $\mathbb{Z}$-градуированности алгебры Ли $\mathfrak{n}$ следует, что $X \in \mathfrak{v}_{-}$сохраняет $\mathcal{D}$ тогда и только тогда, когда $X$ коммутирует со всеми $Y_{i}$, где $i \in I_{1}$, а значит, и со всеми $Y_{i}$, где $i \in I$, поскольку $\mathfrak{n}_{-1}$ порождает $\mathfrak{n}$.

Следовательно, $\mathfrak{g}_{-}$определяется как максимальная подалгебра алгебры $\mathfrak{v}_{-}$, сохраняющая распределение $\mathcal{D}$. Но тогда полное продолжение алгебры $\mathfrak{g}_{-}$является максимальной подалгеброй алгебры всех векторных полей $\mathfrak{v e c t}(n), \operatorname{coxpaняющей~}$ распределение $\mathcal{D}$.

Конечно, мы можем переформулировать все это и без обращения к группе $N$. Нам нужен лишь централизатор $\mathfrak{c e n t}_{\mathfrak{v}_{-}}\left(\mathfrak{g}_{-}\right)$алгебры $\mathfrak{g}_{-}$в алгебре $\mathfrak{v}_{-}$. Ясно также, что, представив поле $Y \in \mathfrak{v}_{-s}$ в виде суммы однородных в смысле стандартной градуировки $\left(\operatorname{deg} x^{i}=1\right)$ компонент,

$$
Y=\sum_{p=-1}^{d-s-1} Y_{(p)},
$$

мы получим, что для полей $Y$, обращающихся в нуль в нуле (для них $\left.Y_{(-1)}=0\right)$, младшая компонента коммутатора $\left[X_{i}, Y\right]$ совпадает с коммутатором младшей компоненты поля $Y$ с $\partial_{i}$ и, следовательно, отлична от нуля. 
Обратно, для любого поля $Y$ такого, что $Y_{(-1)} \neq 0$, уравнения $\left[X_{i}, Y\right]=0, i=$ $1, \ldots, n$, позволяют последовательно однозначно восстановить все компоненты $Y_{(p)}$ для $p \geqslant 0$, начиная с $Y_{(-1)}$, если использовать рекуррентное соотношение

$$
\left[\partial_{i}, Y_{(p)}\right]=-\sum_{s=-1}^{p-1}\left[\left(X_{i}\right)_{(p-1-s)}, Y_{(s)}\right], \quad i=1, \ldots, n
$$

Пусть $Y_{i} \in \mathfrak{c e n t}_{\mathfrak{v}_{-}}\left(\mathfrak{g}_{-}\right)$такое поле, что $\left(Y_{i}\right)_{(-1)}=\partial_{i}$. Тогда

$$
\begin{aligned}
{\left[Y_{i}, Y_{j}\right]_{(-1)} } & =\left[\partial_{i},\left(Y_{j}\right)_{(0)}\right]+\left[\left(Y_{i}\right)_{(0)}, \partial_{j}\right]=-\left[\left(X_{i}\right)_{(0)}, \partial_{j}\right]-\left[\partial_{i},\left(X_{j}\right)_{(0)}\right]= \\
& =-\left[X_{i}, X_{j}\right]_{(-1)}=-\sum_{k} c_{i j}^{k}\left(X_{k}\right)_{(-1)}=-\sum_{k} c_{i j}^{k}\left(Y_{k}\right)_{(-1)},
\end{aligned}
$$

и, поскольку поля из централизатора $\mathfrak{c e n t}_{\mathfrak{v}_{-}}\left(\mathfrak{g}_{-}\right)$однозначно определяются своей (-1)-й компонентой, мы получаем, что

$$
\left[Y_{i}, Y_{j}\right]=-\sum_{k} c_{i j}^{k} Y_{k}
$$

т.е. централизатор $\mathfrak{c e n t}_{\mathfrak{v}_{-}}\left(\mathfrak{g}_{-}\right)$изоморфен $\mathfrak{n}$.

Пусть $\theta^{i}$ образуют базис 1-форм, двойственный базису $\left\{Y_{i}\right\}_{i \in I}$ (т.е. $\left.\theta^{i}\left(Y_{j}\right)=\delta_{j}^{i}\right)$. Тогда любое векторное поле $X \in \mathfrak{v e c t}(n)$ можно представить в виде

$$
X=\sum_{i} \theta^{i}(X) Y_{i}
$$

Поскольку $\left[X_{i}, Y_{j}\right]=0$ для любых $i, j=1, \ldots, n$, мы получаем следующее соотношение:

$$
\theta^{i}\left(\left[X_{j}, X\right]\right)=X_{j}\left(\theta^{i}(X)\right) .
$$

Рассмотрим теперь распределение $\mathcal{D}$, определенное соотношениями (10). Как мы уже заметили, подалгебра $\mathfrak{g}_{-}$характеризуется как максимальная подалгебра алгебры $\mathfrak{v}_{-}$, сохраняющая распределение $\mathcal{D}$.

Заметим прежде всего, что, хотя поле $X_{i}$ сохраняет любую форму $\theta^{j}$, это условие не выдерживает операции (2) полного продолжения. С другой стороны, требование того, что поле $X$ сохраняет распределение $\mathcal{D}$, не является столь сильным и выдерживает продолжение.

Действительно, поле $X \in \mathfrak{v}$ сохраняет $\mathcal{D}$ тогда и только тогда, когда

$$
\theta^{k}\left(\left[X, Y_{i}\right]\right)=0 \quad \text { для любых } i=1, \ldots, n_{1} \text { и любого } k>n_{1} .
$$

Пусть это соотношение выполнено для любого поля $X \in \mathfrak{g}_{s-1}$. Тогда в силу условия (2) $X \in \mathfrak{g}_{s}$ тогда и только тогда, когда

$$
\begin{array}{ll}
\theta^{k}\left(\left[\left[X_{j}, X\right], Y_{i}\right]\right)=X_{j} \theta^{k}\left(\left[X, Y_{i}\right]\right)=0 \quad & \text { для любых } i, j=1, \ldots, n_{1} \\
& \text { и любого } k>n_{1}
\end{array}
$$

(мы учли равенство (13)). 
Наконец, поскольку компонента $\mathfrak{n}_{-1}$ порождает алгебру $\mathfrak{n}$, условие (15) эквивалентно условию

$$
\partial_{j}\left(\theta^{k}\left(\left[X, Y_{i}\right]\right)=0 \quad \text { для всех } j=1, \ldots, n .\right.
$$

Если $k \in I_{l} \quad(l \geqslant 2)$, то $\theta^{k}\left(\left[X, Y_{i}\right]\right)$ является однородным (в нестандартной градуировке) многочленом степени $s-1+l \geqslant s+1 \geqslant 1$, следовательно, условие (16) эквивалентно условию

$$
\theta^{k}\left(\left[X, Y_{i}\right]\right)=0 \quad \text { для любых } i=1, \ldots, n_{1} \text { и любого } k>n_{1},
$$

и поэтому поле $X$ сохраняет распределение $\mathcal{D}$.

Перепишем систему (14) в координатах поля $X$ более подробно:

$$
Y_{i}\left(\theta^{k}(X)\right)-\sum_{j}(-1)^{p\left(Y_{i}\right) p\left(\theta^{j}(X)\right)} c_{i j}^{k} \theta^{j}(X)=0, \quad i=1, \ldots, n_{1}, k=n_{1}+1, \ldots, n .
$$

Градуированность алгебры $\mathfrak{n}$ означает, что эта система имеет специальный вид. Обозначим через $F_{i}=\theta^{n-n_{d}+i}(X), i=1, \ldots, n_{d}$, координаты векторного поля $X$, соответствующие компоненте $\mathfrak{g}_{-d}$ максимальной глубины. Если функции $F_{i}$ известны, то уравнения (18) при $k \in I_{d}$ задают систему линейных (не дифференциальных) уравнений на координаты $\theta^{j}(X)$, отвечающие компоненте $\mathfrak{g}_{-d+1}$, причем если в этой компоненте нет элементов центра всей алгебры $\mathfrak{g}_{-}$, то в систему входят все координаты этого уровня. После того как эти координаты определены, уравнения (18) при $k \in I_{d-1}$ превращаются в систему линейных уравнений на координаты предыдущего уровня $-d+2$ и т.д. Таким образом, функции $F_{i}$ являются производящими функциями для векторного поля $X$. В общем случае в качестве производящих функций следует взять координаты, соответствующие всем линейно независимым центральным элементам алгебры $\mathfrak{g}_{-}$.

Теперь мы в состоянии сформулировать алгоритм для решения первых двух задач.

\section{2. АЛГОРИТМ РЕШЕНИЯ ЗАДАЧ 1, 2}

Мы предлагаем следующий алгоритм.

1. Выбираем в алгебре $\mathfrak{n}$ базис $B$, согласованный с градуировкой и вычисляем соответствующие ему структурные константы $c_{i j}^{k}$.

2. Ищем базис 1-форм $\left\{\omega^{i}\right\}_{i \in I}$, удовлетворяющий уравнениям (4): решаем систему (6) снизу вверх, начиная со степени 1 и поднимаясь последовательно до степени $d$.

3. Ищем двойственный базис векторных полей $\left\{X_{i}\right\}_{i \in I}$ снизу вверх, начиная со степени $-d$ и поднимаясь последовательно до степени -1 . Поля $\left\{X_{i}\right\}_{i \in I}$ определяют вложение алгебры $\mathfrak{n}$ в алгебру $\mathfrak{v e c t}(n)$. Задача 1 решена.

4. Ищем базис $\left\{Y_{i}\right\}_{i \in I}$ централизатора $\mathfrak{c e n t}_{\mathfrak{v}_{-}}\left(\mathfrak{g}_{-}\right)$в алгебре $\mathfrak{v}_{-}$с помощью соотношений (11) и двойственный ему базис 1-форм $\left\{\theta^{i}\right\}_{i \in I}$.

5. Для того чтобы найти компоненту $\mathfrak{g}_{s}$ полного продолжения алгебры $\mathfrak{g}_{-}$, ищем поле $X \in \mathfrak{g}_{s}$ в виде $X=\sum \theta^{i}(X) Y_{i}$. Для этого представляем каждую из $n_{d}$ производящих функций $F^{i}=\theta^{n-n_{d}+i}(X)$ в виде суммы мономов степени $d+s$ (в нестандартной градуировке) с неопределенными коэффициентами и решаем систему (18) линейных уравнений на эти коэффициенты. Для контроля при $s<0$ сверяем вид получающихся полей с полями $X_{i}$. Задача 2 решена. 
ПримеР. Пусть всюду в дальнейшем в зависимости от характеристики поля $p$

$$
x^{(k)}:= \begin{cases}x^{k} / k !, & p=0 \\ u^{(k)}, & p>0\end{cases}
$$

где $u^{(k)}-$ разделенная степень.

Пусть $\mathfrak{n}=\mathfrak{n}_{-3} \oplus \mathfrak{n}_{-2} \oplus \mathfrak{n}_{-1}$ обозначает единственную $\mathbb{Z}$-градуированную нильпотентную алгебру Ли, удовлетворяющую условию (1), такую, что

$$
\operatorname{dim} \mathfrak{n}_{-1}=2, \quad \operatorname{dim} \mathfrak{n}_{-2}=1, \quad \operatorname{dim} \mathfrak{n}_{-3}=2 .
$$

Как отмечено в [1], эта алгебра тесно связана с простой исключительной алгеброй Ли $\mathfrak{g}(2)$. Если на алгебре Ли $\mathfrak{g}(2)$ ввести $\mathbb{Z}$-градуировку, положив степень длинного простого корня равной нулю, а степень короткого простого корня - равной единице, то в разложении $\mathfrak{g}(2)=\mathfrak{g}(2)_{-} \oplus \mathfrak{g}(2)_{0} \oplus \mathfrak{g}(2)_{+}$градуированная подалгебра $\mathfrak{g}(2)_{-}$ изоморфна $\mathfrak{n}$, причем, как показано в [5], полное продолжение алгебры $\mathfrak{n}$ изоморфно в точности исключительной алгебре Ли $\mathfrak{g}(2)$.

Проследим действие алгоритма на примере вложения $f(\mathfrak{n})=\mathfrak{g}_{-} \subset \mathfrak{v e c t}(5)$.

1. Базис, согласованный с градуировкой, и структурные константы имеют вид

$$
\begin{gathered}
\mathfrak{n}_{-1}=\operatorname{Span}\left(e_{1}, e_{2}\right), \quad \mathfrak{n}_{-2}=\operatorname{Span}\left(e_{3}\right), \quad \mathfrak{n}_{-3}=\operatorname{Span}\left(e_{4}, e_{5}\right) \\
{\left[e_{1}, e_{2}\right]=e_{3}, \quad\left[e_{1}, e_{3}\right]=e_{4}, \quad\left[e_{2}, e_{3}\right]=e_{5}}
\end{gathered}
$$

$c_{i j}^{k}=0 \quad$ при $k=1,2, \quad c_{12}^{3}=-c_{21}^{3}=1, \quad c_{i j}^{3}=0 \quad$ в остальных случаях;

$c_{13}^{4}=-c_{31}^{4}=1, \quad c_{i j}^{4}=0 \quad$ в остальных случаях;

$c_{23}^{5}=-c_{32}^{5}=1, \quad c_{i j}^{5}=0 \quad$ в остальных случаях.

2. Тогда

$$
\begin{aligned}
& \omega^{1}=d x^{1}, \quad \omega^{2}=d x^{2} \Longrightarrow \quad V_{i}^{k}=\delta_{i}^{k} \text { при } k=1,2 ; \\
& \omega^{3}=d x^{3}+\sum_{i, j=1}^{2} a_{i j}^{3} x^{i} d x^{j} \Longrightarrow \quad V_{4}^{3}=V_{5}^{3}=0, \quad V_{3}^{3}=1 ; \\
& V_{2}^{3}=a_{12}^{3} x^{1}+a_{22}^{3} x^{2}, \quad V_{1}^{3}=a_{11}^{3} x^{1}+a_{21}^{3} x^{2} .
\end{aligned}
$$

Уравнения (6) дают одно нетривиальное соотношение на функции $V_{i}^{3}$ :

$$
\partial_{2} V_{1}^{3}-\partial_{1} V_{2}^{3}=V_{1}^{1} V_{2}^{2}-V_{1}^{2} V_{2}^{1}=1,
$$

или (в коэффициентах $a_{i j}^{3}$ )

$$
a_{21}^{3}-a_{12}^{3}=1
$$

В сущности, мы можем взять любой набор коэффициентов $a_{11}^{3}, a_{22}^{3}, a_{12}^{3}, a_{21}^{3}$, удовлетворяющий (19). Возьмем тот, который кажется наиболее простым:

$$
a_{11}^{3}=a_{22}^{3}=a_{12}^{3}=0, \quad a_{21}^{3}=1 .
$$


В канонических координатах первого рода $a_{11}^{3}=a_{22}^{3}=0, a_{12}^{3}=a_{21}^{3}=1 / 2$, и это наиболее симметричные координаты. Однако сейчас нам хочется по возможности избежать делений.

Таким образом, $V_{1}^{3}=x^{2}, V_{2}^{3}=0$, и следовательно,

$$
\omega^{3}=d x^{3}+x^{2} d x^{1} .
$$

Далее, при $k=4,5$

$$
\omega^{k}=d x^{k}+\sum_{j=1}^{3} V_{j}^{k} d x^{j},
$$

где

$$
\begin{aligned}
& V_{3}^{k}=a_{1}^{k} x^{1}+a_{2}^{k} x^{2}, \\
& V_{j}^{k}=\alpha_{j}^{k}\left(x^{1}\right)^{(2)}+\beta_{j}^{k} x^{1} x^{2}+\gamma_{j}^{k}\left(x^{2}\right)^{(2)}+\varepsilon_{j}^{k} x^{3}, \quad j=1,2 .
\end{aligned}
$$

Уравнения (6) дают по три нетривиальных соотношения на функции $V_{j}^{k}$, где $k=4,5$, $j=1,2,3$ :

$$
\begin{array}{lll}
\partial_{2} V_{1}^{4}-\partial_{1} V_{2}^{4}=0, & \partial_{3} V_{1}^{4}-\partial_{1} V_{3}^{4}=1, & \partial_{3} V_{2}^{4}-\partial_{2} V_{3}^{4}=0 ; \\
\partial_{2} V_{1}^{5}-\partial_{1} V_{2}^{5}=-x^{2}, & \partial_{3} V_{1}^{5}-\partial_{1} V_{3}^{5}=0, & \partial_{3} V_{2}^{5}-\partial_{2} V_{3}^{5}=1 ;
\end{array}
$$

или (в терминах коэффициентов):

$$
\begin{array}{lll}
\beta_{1}^{4} x^{1}+\gamma_{1}^{4} x^{2}=\alpha_{2}^{4} x^{1}+\beta_{2}^{4} x^{2}, & \varepsilon_{1}^{4}-a_{1}^{4}=1, & \varepsilon_{2}^{4}-a_{2}^{4}=0 ; \\
\beta_{1}^{5} x^{1}-\gamma_{1}^{5} x^{2}-\alpha_{2}^{5} x^{1}-\beta_{2}^{5} x^{2}=-x^{2}, & \varepsilon_{1}^{5}-a_{1}^{5}=1, & \varepsilon_{2}^{5}-a_{2}^{5}=1 .
\end{array}
$$

Выбирая наиболее простое решение, получим

$$
\omega^{4}=d x^{4}-x^{1} d x^{3}, \quad \omega^{5}=d x^{5}-x^{2} d x^{3}-\left(x^{2}\right)^{(2)} d x^{1} .
$$

Итак,

$$
\begin{aligned}
& \omega^{1}=d x^{1}, \quad \omega^{2}=d x^{2}, \quad \omega^{3}=d x^{3}+x^{2} d x^{1}, \\
& \omega^{4}=d x^{4}-x^{1} d x^{3}, \quad \omega^{5}=d x^{5}-x^{2} d x^{3}-\left(x^{2}\right)^{(2)} d x^{1} .
\end{aligned}
$$

3. Теперь ищем двойственные поля $X_{i}$ :

$$
\begin{array}{ll}
X_{5}=\partial_{5}, & X_{4}=\partial_{4}, \quad X_{3}=\partial_{3}+x^{1} \partial_{4}+x^{2} \partial_{5}, \\
X_{2}=\partial_{2}, & X_{1}=\partial_{1}-x^{2} \partial_{3}-x^{1} x^{2} \partial_{4}-\left(x^{2}\right)^{(2)} \partial_{5} .
\end{array}
$$

Получаем $\mathfrak{g}_{-}=\operatorname{Span}\left\{X_{1}, \ldots, X_{5}\right\}$.

4. Ищем однородные поля $Y_{i}=\partial_{i}+\cdots$, коммутирующие со всеми $X_{j}$. Коммутирование с $X_{2}, X_{4}, X_{5}$ означает, что все координаты полей $Y_{i}$ могут зависеть только от переменных $x^{1}$ и $x^{3}$. Тогда

$$
Y_{4}=\partial_{4}, \quad Y_{5}=\partial_{5}, \quad Y_{3}=\partial_{3}+a x^{1} \partial_{4}+b x^{1} \partial_{5},
$$

и из равенства $\left[X_{1}, Y_{3}\right]=0$ следует, что $Y_{3}=\partial_{3}$. 
Наконец, при $i=1,2$ поля $Y_{i}$ имеют вид

$$
Y_{i}=\partial_{i}+\alpha_{i} x^{1} \partial_{3}+\sum_{j=4}^{5}\left(\beta_{i}^{j}\left(x^{1}\right)^{(2)}+\gamma_{i}^{j} x^{3}\right) \partial_{j} .
$$

Коммутируя $Y_{i}$ с $X_{1}$ и $X_{3}$, получаем

$$
\begin{aligned}
& Y_{1}=\partial_{1}+x^{3} \partial_{4} \\
& Y_{2}=\partial_{2}-x^{1} \partial_{3}-\left(x^{1}\right)^{(2)} \partial_{4}+x^{3} \partial_{5} .
\end{aligned}
$$

Осталось только найти формы $\theta^{i}$, двойственные полям $Y_{i}$. Стандартные вычисления показывают, что $\theta^{i}=d x^{i}$ при $i=1,2$ и

$$
\begin{aligned}
& \theta^{3}=d x^{3}+x^{1} d x^{2}, \\
& \theta^{4}=d x^{4}-x^{3} d x^{1}+\left(x^{1}\right)^{(2)} d x^{2}, \\
& \theta^{5}=d x^{5}-x^{3} d x^{2} .
\end{aligned}
$$

5. Ищем все векторные поля $X$, сохраняющие распределение $\mathcal{D}=\operatorname{Span}\left\{Y_{1}, Y_{2}\right\}$, или, что то же самое, все поля, принадлежащие полному продолжению алгебры Ли $\mathfrak{g}_{-}$. Поле $X$ ищем в виде $X=\sum f^{i} Y_{i}$, где $f^{i}=\theta^{i}(X)$. Для этого решаем уравнения (18). В нашем случае они имеют вид

$$
\begin{array}{lll}
Y_{1}\left(f^{4}\right)=f^{3}, & Y_{1}\left(f^{5}\right)=0, & Y_{2}\left(f^{4}\right)=0, \\
Y_{2}\left(f^{5}\right)=f^{3}, & Y_{1}\left(f^{3}\right)=f^{2}, & Y_{2}\left(f^{3}\right)=-f^{1} .
\end{array}
$$

Мы видим, что векторное поле $X$ полностью определяется компонентами $f^{4}$ и $f^{5}$, для которых имеются три соотношения

$$
Y_{1}\left(f^{5}\right)=0, \quad Y_{2}\left(f^{4}\right)=0, \quad Y_{1}\left(f^{4}\right)=Y_{2}\left(f^{5}\right) .
$$

Для контроля мы можем посмотреть, какие поля выделяют эти формулы в компоненте $\mathfrak{v}_{-2}$. Обе функции $f^{4}$ и $f^{5}$ должны иметь степень единица в нашей градуировке, т.е. иметь вид $f^{i}=a^{i} x^{1}+b^{i} x^{2}, i=4,5$. Тогда $Y_{1}\left(f^{i}\right)=a, Y_{2}\left(f^{i}\right)=b$, и уравнения (22) означают, что

$$
f^{4}=a x^{1}, \quad f^{5}=a x^{2} \Longrightarrow f^{3}=a, \quad f^{1}=f^{2}=0 .
$$

Таким образом, любое поле, сохраняющее распределение $\mathcal{D}$ и лежащее в компоненте $\mathfrak{v}_{-2}$, пропорционально полю

$$
X=Y_{3}+x^{1} Y_{4}+x^{2} Y_{5}=\partial_{3}+x^{1} \partial_{4}+x^{2} \partial_{5}=X_{3},
$$

в чем мы и хотели убедиться.

Аналогично мы можем убедиться в том, что и в компоненте $\mathfrak{v}_{-1}$ наши уравнения выделяют в точности подпространство, натянутое на поля $X_{1}$ и $X_{2}$.

Перейдем к вычислению нулевой компоненты. Производящие функции для нее должны иметь степень три в нашей градуировке, т.е. иметь вид (для $i=4,5$ )

$$
f^{i}=a_{1}^{i}\left(x^{1}\right)^{(3)}+a_{2}^{i}\left(x^{1}\right)^{(2)} x^{2}+a_{3}^{i} x^{1}\left(x^{2}\right)^{(2)}+a_{4}^{i}\left(x^{2}\right)^{(3)}+b_{1}^{i} x^{1} x^{3}+b_{2}^{i} x^{2} x^{3}+c_{1}^{i} x^{4}+c_{2}^{i} x^{5} .
$$


Тогда

$$
\begin{aligned}
& Y_{1}\left(f^{i}\right)=a_{1}^{i}\left(x^{1}\right)^{(2)}+a_{2}^{i} x^{1} x^{2}+a_{3}^{i}\left(x^{2}\right)^{(2)}+\left(b_{1}^{i}+c_{1}^{i}\right) x^{3}, \\
& Y_{2}\left(f^{i}\right)=\left(a_{2}^{i}-2 b_{1}^{i}-c_{1}^{i}\right)\left(x^{1}\right)^{(2)}+\left(a_{3}^{i}-b_{2}^{i}\right) x^{1} x^{2}+a_{4}^{i}\left(x^{2}\right)^{(2)}+\left(b_{2}^{i}+c_{2}^{i}\right) x^{3} .
\end{aligned}
$$

Уравнения (22) в этом случае сводятся к следующей системе линейных уравнений:

$$
\begin{gathered}
a_{1}^{5}=a_{2}^{5}=a_{3}^{5}=0, \quad b_{1}^{5}+c_{1}^{5}=0, \\
a_{4}^{4}=0, \quad b_{2}^{4}+c_{2}^{4}=0, \quad a_{3}^{4}-b_{2}^{4}=0, \quad a_{2}^{4}-2 b_{1}^{4}+c_{1}^{4}=0, \\
a_{1}^{4}=a_{2}^{5}-2 b_{1}^{5}-c_{1}^{5}, \quad a_{2}^{4}=a_{3}^{5}-b_{2}^{5}, \quad a_{3}^{4}=a_{4}^{5}, \quad b_{1}^{4}+c_{1}^{4}=b_{2}^{5}+c_{2}^{5} .
\end{gathered}
$$

Решая эту систему, получаем

$$
\begin{array}{ll}
a_{1}^{4}=-b_{1}^{5}=c_{1}^{5}=\alpha, & a_{2}^{4}=-b_{2}^{5}=\beta, \\
a_{3}^{4}=a_{4}^{5}=b_{2}^{4}=-c_{2}^{4}=\gamma, & a_{4}^{4}=a_{1}^{5}=a_{2}^{5}=a_{3}^{5}=0, \\
b_{1}^{4}=\delta, \quad c_{1}^{4}=\beta-2 \delta, & c_{2}^{5}=2 \beta-\delta .
\end{array}
$$

Откуда

$$
\begin{aligned}
& f^{5}=\gamma\left(x^{2}\right)^{3}-\alpha x^{1} x^{3}-\beta x^{2} x^{3}+\alpha x^{4}+(2 \beta-\delta) x^{5} \\
& f^{4}=\alpha\left(x^{1}\right)^{(3)}+\beta\left(x^{1}\right)^{(2)} x^{2}+\gamma x^{1}\left(x^{2}\right)^{(2)}+\delta x^{1} x^{3}+\gamma x^{2} x^{3}+(\beta-2 \delta) x^{4}-\gamma x^{5}, \\
& f^{3}=\alpha\left(x^{1}\right)^{(2)}+\beta x^{1} x^{2}+\gamma\left(x^{2}\right)^{(2)}+(\beta-\delta) x^{3} \\
& f^{2}=Y_{1}\left(f^{3}\right)=\alpha x^{1}+\beta x^{2}, \quad f^{1}=-Y_{2}\left(f^{3}\right)=-\delta x^{1}-\gamma x^{2} .
\end{aligned}
$$

В качестве базиса компоненты $\mathfrak{g}_{0}$ возьмем векторы $X_{\alpha}, X_{\beta}, X_{\gamma}, X_{\delta}$, отвечающие только одному ненулевому параметру (например, $X_{\alpha}$ отвечает значениям $\alpha=1$, $\beta=\gamma=\delta=0$ и т.д.):

$$
\begin{aligned}
X_{\alpha} & =x^{1} Y_{2}+\left(x^{1}\right)^{(2)} Y_{3}+\left(x^{1}\right)^{(3)} Y_{4}+\left(-x^{1} x^{3}+x^{4}\right) Y_{5}= \\
& =x^{1} \partial_{2}+x^{4} \partial_{5}-\left(x^{1}\right)^{(2)} \partial_{3}-2\left(x^{1}\right)^{(2)} \partial_{4}, \\
X_{\beta} & =x^{2} \partial_{2}+x^{3} \partial_{3}+x^{4} \partial_{4}+2 x^{5} \partial_{5}, \\
X_{\gamma} & =-x^{2} \partial_{1}-x^{5} \partial_{4}+\left(x^{2}\right)^{(2)} \partial_{3}+x^{1}\left(x^{2}\right)^{(2)} \partial_{4}+\left(x^{2}\right)^{(3)} \partial_{5}, \\
X_{\delta} & =-x^{1} \partial_{1}-x^{3} \partial_{3}-2 x^{4} \partial_{4}-x^{5} \partial_{5} .
\end{aligned}
$$

При $\alpha=\gamma=0, \quad \delta=-\beta=1$, получаем градуирующий оператор

$$
X=-x^{1} \partial_{1}-x^{2} \partial_{2}-2 x^{3} \partial_{3}-3 x^{4} \partial_{4}-3 x^{5} \partial_{5} .
$$

Высшие компоненты вычисляются аналогичным образом.

Интерпретация результатов. Существуют три реализации алгебры $\mathfrak{g}=\mathfrak{g}(2)$ в виде алгебры Ли векторных полей, сохраняющих некоторое неинтегрируемое распределение на $\mathfrak{g}_{-}$. Эти реализации связаны с тремя (несжимаемыми) $\mathbb{Z}$-градуировками алгебры $\mathfrak{g}:$ степень одного или обоих корневых векторов полагается равной 
единице. Выше мы рассмотрели градуировку $(1,0)$; Картан использовал ее для того, чтобы дать первую интерпретацию алгебры $\mathfrak{g}(2)$, в то время только что открытой Киллингом, см. [1 $]^{5)}$.

В нашей реализации (полями $X_{i}$ ) алгебра $\mathfrak{g}=\mathfrak{g}(2)$ сохраняет распределение в касательном расслоении на $\mathfrak{g}_{-}$, задаваемое системой уравнений Пфаффа на векторные поля $X$

$$
\theta^{3}(X)=0, \quad \theta^{4}(X)=0, \quad \theta^{5}(X)=0 .
$$

Эквивалентным, но чуть более экономным способом можно описать алгебру $\mathfrak{g}=$ $\mathfrak{g}(2)$ как алгебру, сохраняющую кораспределение в кокасательном расслоении на $\mathfrak{g}_{-}$, задаваемое векторами (20), т.е. следующей системой уравнений на 1-формы $\alpha$ :

$$
\alpha\left(Y_{1}\right)=0, \quad \alpha\left(Y_{2}\right)=0 .
$$

Очевидно, что описание в терминах кораспределений иногда оказывается короче: любое распределение коразмерности $r$ описывается с помощью $r$ уравнений Пфаффа, тогда как двойственное кораспределение - с помощью $n-r$ уравнений.

Аналогично можно описать и остальные реализации алгебры $\mathfrak{g}(2)$, отвечающие другим $\mathbb{Z}$-градуировкам, различные реализации исключительных алгебр Ли $\mathfrak{f}(4)$ и $\mathfrak{e}(6)-\mathfrak{e}(8)$, а также исключительные супералгебры Ли и алгебры Ли над полями характеристики $p>0$. До появления работы [2] рассматривать неинтегрируемые распределения, связанные с различными $\mathbb{Z}$-градуировками неисключительных алгебр Ли, представлялось ненужным: их обычное описание как сохраняющих объем или невырожденную билинейную форму достаточно ясно. Картан, хотя и отмечал важность описания алгебр Ли в терминах распределений, рассматривал для каждой конкретной алгебры Ли только одно-два распределения, связанных с одной-двумя ее $\mathbb{Z}$-градуировками. Однако если применить описанный алгоритм к алгебрам o(7), $\mathfrak{s p}(4)$ и $\mathfrak{s p}(10)$ при характеристике $p=2,3,5$, то удается прояснить строение некоторых простых алгебр Ли, специфических для этих характеристик, и получить некоторые новые примеры, как в [2], необходимые для будущей классификации.

\section{3. КАК ВЫДЕЛЯТЬ ЧАСТИЧНЫЕ ПРОДОЛЖЕНИЯ: РЕШЕНИЕ ЗАДАЧИ 3}

Итак, мы описали полное продолжение (супер)алгебры Ли $\mathfrak{g}_{-}$, т.е. нам известна максимальная подалгебра $\mathfrak{g}=\left(\mathfrak{g}_{-}\right)_{*} \subset \mathfrak{v}$ с данной отрицательной частью. Рассмотрим теперь подпространство $\mathfrak{h}=\mathfrak{g}_{-} \oplus\left(\bigoplus_{0 \leqslant k \leqslant K} \mathfrak{h}_{k}\right) \subset \mathfrak{g}$, замкнутое относительно скобки в пределах своей градуировки, другими словами, такое, что $\left[\mathfrak{h}_{i}, \mathfrak{h}_{j}\right] \subset \mathfrak{h}_{i+j}$, если только $i+j \leqslant K$. Опишем частичное продолжение $\mathfrak{h}_{*}=\bigoplus_{k \geqslant-d} \mathfrak{h}_{k} \subset \mathfrak{g}$ подпространства $\mathfrak{h}$, т.е. максимальную подалгебру алгебры $\mathfrak{g}$ с данной начальной частъю $\mathfrak{h}$. Компоненты $\mathfrak{h}_{k}$ с $k>K$ выделяются условием (2). При этом под описанием мы имеем в виду выделение подалгебры $\mathfrak{h}$ в $\mathfrak{g}$ с помощью системы дифференциальных уравнений.

5) Картан рассматривал также градуировку $(1,1)$, см. [5]; именно в такой градуировке алгебра $\mathfrak{g}(2)$ появилась у Картана при изучении уравнения Гильберта $f^{\prime}=\left(g^{\prime \prime}\right)^{2}$. Наконец, градуировка $(0,1)$ была недавно рассмотрена в [9]. 
ЗАмечАниЕ. Картановское продолжение $\left(\mathfrak{g}_{-}, \mathfrak{g}_{0}\right)_{*}$, где подалгебра $\mathfrak{g}_{-}$коммутативна, глубина $d=1$, а $\mathfrak{g}_{0} \subset \mathfrak{g l}(n)$, является частным случаем вышеописанной конструкции при $\mathfrak{g}=\mathfrak{v e c t}(n)$ и $\mathfrak{h}=\mathfrak{g}_{-} \oplus \mathfrak{g}_{0}$. Примеры описания картановских продолжений с помощью дифференциальных уравнений см. в [6], [7].

Будем называть однородную компоненту $\mathfrak{h}_{m}$ алгебры $\mathfrak{h}$ определяющей, если $\mathfrak{h}_{k}=$ $\mathfrak{g}_{k}$ для всех $k<m$, но $\mathfrak{h}_{m} \neq \mathfrak{g}_{m}$. Мы рассмотрим алгоритм описания алгебры $\mathfrak{h}_{*}$ для случая, когда определяющая компонента имеет максимальную степень $K$. Случай, когда определяющая компонента имеет степень $m<K$, сводится к предыдущему. Действительно, мы можем сначала описать частичное продолжение $\mathfrak{h}_{*}^{m}=$ $\left(\bigoplus_{0 \leqslant k \leqslant m} \mathfrak{h}_{k}\right)_{*}$, сравнить компоненты $\mathfrak{h}_{k}$ при $m<k \leqslant K$ с соответствующими компонентами полученного продолжения $\mathfrak{h}_{*}^{m}$, выделить новую определяющую компоненту, если она появится, и т.д.

Итак, пусть $Z_{1}, \ldots, Z_{\operatorname{dim} \mathfrak{h}_{K}}-$ базис определяющей компоненты $\mathfrak{h}_{K} \subset \mathfrak{g}_{K}$.

Прежде всего, мы должны выделить подпространство $\mathfrak{h}_{K}$ в $\mathfrak{g}_{K}$ с помощью системы линейных (алгебраических) уравнений, т.е. найти базис аннулятора подпространства $\mathfrak{h}_{K}$ в $\left(\mathfrak{g}_{K}\right)^{*}$, или, что эквивалентно, найти фундаментальную систему решений $\alpha^{1}, \ldots, \alpha^{r}$ следующей системы уравнений на неизвестные 1 -формы $\alpha \in\left(\mathfrak{g}_{K}\right)^{*}$ :

$$
\alpha\left(Z_{i}\right)=0, \quad i=1, \ldots, \operatorname{dim} \mathfrak{h}_{K} .
$$

Подпространство $\mathfrak{h}_{K}$ выделяется с помощью системы однородных линейных уравнений на неизвестное векторное поле $X \in \mathfrak{g}_{K}$

$$
\alpha^{i}(X)=0, \quad i=1, \ldots, r .
$$

В компоненте $\mathfrak{g}_{K}$ есть удобный для нас базис, состоящий из полей вида $f Y_{j}$, где $f-$ моном степени $K+s$, если $j \in I_{s}$. Соответственно, двойственный базис состоит из элементов степени $-K$ и имеет вид $A_{i_{1}, \ldots, i_{t}}^{j}=S\left(Y_{i_{1}} \ldots Y_{i_{t}}\right) \theta^{j}$, а формы $\alpha^{l}$ могут быть представлены в этом базисе как

$$
\alpha^{l}=\sum a_{j}^{l ; i_{1}, \ldots, i_{t}} A_{i_{1}, \ldots, i_{t}}^{j} .
$$

Подставляя (25) в (24), получим следующую систему однородных линейных дифференциальных уравнений с постоянными коэффициентами на координаты векторного поля $X=\theta^{i}(X) Y_{i} \in \mathfrak{h}_{K}$ :

$$
\sum a_{j}^{l ; i_{1}, \ldots, i_{t}} S\left(Y_{i_{1}} \ldots Y_{i_{t}}\right) \theta^{j}(X)=0, \quad l=1, \ldots, r .
$$

Заметим, что для алгебр Ли (но не для супералгебр Ли) уравнения (26) выдерживают процедуру продолжения (2). Действительно, для $k>K$, по предположению индукции, $X \in \mathfrak{h}_{k}$ тогда и только тогда, когда коммутаторы $\left[X_{i}, X\right]$ удовлетворяют уравнениям (26) для всех $i=1, \ldots, n_{1}$. Положим

$$
f^{l}=\sum a_{j}^{l ; i_{1}, \ldots, i_{t}} S\left(Y_{i_{1}} \ldots Y_{i_{t}}\right) \theta^{j}(X) .
$$

Поскольку все поля $X_{i}$ коммутируют со всеми полями $Y_{j}$, система $(26)$, в которую вместо поля $X$ подставлены коммутаторы $\left[X_{i}, X\right]$, эквивалентна системе

$$
X_{i}\left(f^{l}\right)=0, \quad i=1, \ldots, n_{1}, \quad l=1, \ldots, r,
$$


которая в свою очередь в силу (1) эквивалентна системе

$$
\partial_{i}\left(f^{l}\right)=0, \quad i=1, \ldots, n, \quad l=1, \ldots, r .
$$

Это означает, что $f^{l}=$ const для всех $l=1, \ldots, r$. Поскольку функции $f^{l}$ являются однородными многочленами степени $k-K>0$, из этого следует, что $f^{l}=0$. Значит, $X \in \mathfrak{h}_{k}$ тогда и только тогда, когда поле $X$ удовлетворяет системе $(26)$.

В суперслучае поля $X_{i}$ и $Y_{j}$ суперкоммутируют, а не коммутируют, что, вообще говоря, не позволяет нам отбросить $X_{i}$ и перейти от системы (26) к системе (27). Ситуацию спасает следующее простое и хорошо известное соображение, которое мы докажем для полноты изложения.

Напомним, что $p(\cdot)$ обозначает функцию четности, a Pty - оператор четности:

$$
\operatorname{Pty}(x)=(-1)^{p(x)} x .
$$

Лемма. Пусть элементы $X, Y \in$ End $V$ суперкоммутируют $u p(Y)=\overline{1}$. Тогда $X u \widehat{Y}=Y$ Pty коммутируют (в обычном смысле), т.е. $X \widehat{Y}=\widehat{Y} X$.

ДокАзАТЕЛЬСтво. Действительно,

$$
\begin{aligned}
X \widehat{Y}(v) & =X Y \operatorname{Pty}(v)=(-1)^{p(v)} X Y(v)= \\
& =(-1)^{p(v)}(-1)^{p(X) p(Y)} Y X(v)=(-1)^{p(v)+p(X)} Y X(v)=\widehat{Y} X(v) .
\end{aligned}
$$

Следовательно, в суперслучае систему (26) следует записывать с помощью операторов $\widehat{Y}_{i}$ вместо $Y_{i}\left(\right.$ если $p\left(Y_{i}\right)=\overline{0}$, мы полагаем $\left.\widehat{Y}_{i}=Y_{i}\right)$.

Наконец, если $d>1$, то любое поле $X \in \mathfrak{g}$ полностью определяется своими производящими функциями $F^{i}$. Поэтому и уравнения $(26)$ достаточно написать только на производящие функции.

ПримеР 1. Глубина $d=1$. Пусть $\mathfrak{g}_{-}=\mathfrak{g}_{-1}$ коммутативна. Тогда $\mathfrak{g}=\left(\mathfrak{g}_{-}\right)_{*}=$ $\mathfrak{v e c t}(n)$ в стандартной $\mathbb{Z}$-градуировке (степень каждой переменной равна единице). Пусть $\mathfrak{g}_{0}=\mathfrak{g l}(n)=\mathfrak{v e c t}(n)_{0}$. Компонента $\mathfrak{v e c t}(n)_{1}$ степени единица состоит, как известно, из двух неприводимых $\mathfrak{g l}(n)$-модулей. Пусть

$$
X=\sum a_{i j}^{k} x^{i} x^{j} \partial_{k}:=\sum f^{k}(x) \partial_{k} .
$$

Тогда эти подмодули имеют вид

$$
\begin{aligned}
\mathfrak{h}_{1(1)} & :=\operatorname{Span}\left\{x^{i} \sum_{j} x^{j} \partial_{j} \mid i=1, \ldots, n\right\}, \\
\mathfrak{h}_{1(2)} & :=\operatorname{Span}\left\{X=\sum_{j=1}^{n} d_{i j}^{k} x^{i} x^{j} \partial_{k} \mid d_{i i}^{i}+\sum_{j} d_{i j}^{j}=0, \quad i=1, \ldots, n\right\} .
\end{aligned}
$$

Выделим частичные продолжения $\mathfrak{h}_{*(i)}=\left(\mathfrak{g}_{-1} \oplus \mathfrak{g l}(n) \oplus \mathfrak{h}_{1(i)}\right)_{*}, \quad i=1,2$, в алгебре $\mathfrak{v e c t}(n)$ с помощью дифференциальных уравнений на функции $f^{k}(x)$, см. (28). В этом случае $X_{i}=Y_{i}=\partial_{i}$.

Условия на компоненту $\mathfrak{h}_{1(2)}$ можно немедленно представить в виде

$$
\sum_{j} \partial_{i} \partial_{j}\left(f^{j}\right)=0, \quad i=1, \ldots, n,
$$


или в эквивалентном виде

$$
\partial_{i}\left(\sum_{j} \frac{\partial f^{j}}{\partial x^{j}}\right)=0, \quad i=1, \ldots, n .
$$

Это в точности система (26) на векторное поле $X \in \mathfrak{h}_{*(2)}$, переписанная в координатной форме. Ее можно переписать в хорошо известном виде

$$
\sum_{j} \frac{\partial f^{j}}{\partial x^{j}}=\operatorname{div} X=\text { const }
$$

Значит, $\mathfrak{h}_{*(2)}=\mathfrak{d e r}(\mathfrak{s v e c t}(n)):=\mathfrak{s v e c t}(n) \oplus \mathbb{K} E$, где $E=\sum x^{i} \partial_{i}$, a $\mathfrak{s v e c t}(n)$ - алгебра Ли бездивергентных векторных полей. Этот результат хорошо известен.

Теперь рассмотрим продолжение $\mathfrak{h}_{*(1)}$, которое, конечно, есть подалгебра $\mathfrak{s l}(n+1)$, вложенная в $\mathfrak{v e c t}(n)$. Представляя поле $X \in \mathfrak{h}_{1(1)}$ в виде

$$
\left(c_{1}\left(x^{1}\right)^{2}+c_{2} x^{1} x^{2}+\cdots+c_{n} x^{1} x^{n}\right) \partial_{1}+\cdots+\left(c_{1} x^{1} x^{n}+c_{2} x^{2} x^{n}+\cdots+c_{n}\left(x^{n}\right)^{2}\right) \partial_{n},
$$

мы сразу видим, что $d_{i j}^{k}=0$, если $i \neq k, j \neq k$, a $d_{k k}^{k}=d_{k i}^{i}$ для всех $i \neq k$. Соответствующая система дифференциальных уравнений есть

$$
\begin{array}{crl}
\frac{\partial^{2} f^{k}}{\partial x^{i} \partial x^{j}} & =0, & i, j \neq k, \\
\frac{1}{2} \frac{\partial^{2} f^{k}}{\left(\partial x^{k}\right)^{2}} & =\frac{\partial^{2} f^{i}}{\partial x^{i} \partial x^{k}}, & i \neq k .
\end{array}
$$

Суперизация. Для супералгебр, как мы уже видели, следует рассматривать композиции полей $Y_{i}=\partial_{i}$ с оператором четности, т.е. вместо частных производных $\partial_{i}$ рассматривать операторы

$$
\nabla_{i}(f):=(-1)^{p(f) p\left(\partial_{i}\right)} \partial_{i}(f) .
$$

Эти операторы $\nabla_{i}$ коммутируют (а не суперкоммутируют) с любым оператором $X_{j}=\partial_{j}$ из компоненты $\mathfrak{g}_{-1}$. Система (31) примет тогда вид

$$
\nabla_{i}\left(\sum_{j} \nabla_{j}\left(f^{j}\right)\right)=0, \quad i=1, \ldots, n,
$$

что, тем не менее, ведет к тому же условию $\operatorname{div} X=$ const. Это еще один способ увидеть, почему в координатном выражении для дивергенции в суперслучае должны появляться некоторые дополнительные знаки: уравнения (31) не выдерживают процедуры продолжения (2).

Учитывая, что

$$
d_{i j}^{k}=-(-1)^{p\left(f^{k}\right)} \frac{\partial^{2} f^{k}}{\partial x^{i} \partial x^{j}}, \quad d_{k k}^{k}=0 \quad \text { для нечетных } x^{k},
$$

заключаем, что второе уравнение в (32) принимает в суперслучае вид

$$
\begin{aligned}
\frac{1}{2} \frac{\partial^{2} f^{k}}{\left(\partial x^{k}\right)^{2}} & =(-1)^{p\left(x^{i}\right) p\left(f^{i}\right)+1} \frac{\partial^{2} f^{i}}{\partial x^{i} \partial x^{k}} \quad \text { при } \quad p\left(x^{i}\right)=\overline{0}, \quad i \neq k ; \\
(-1)^{p\left(x^{j}\right) p\left(f^{j}\right)} \frac{\partial^{2} f^{i}}{\partial x^{j} \partial x^{k}} & =(-1)^{p\left(x^{i}\right) p\left(f^{i}\right)} \frac{\partial^{2} f^{i}}{\partial x^{i} \partial x^{k}} \quad \text { при } \quad p\left(x^{i}\right)=\overline{0}, \quad i, j \neq k .
\end{aligned}
$$

5 Теоретическая и математическая физика, т. 147, № 3, 2006 г. 
Рассмотрим теперь несколько более или менее известных примеров и один новый пример (kas) глубины $>1$. Во всех них супералгебра Ли $\mathfrak{n}=\mathfrak{n}_{-1} \oplus \mathfrak{n}_{-2}, \quad \operatorname{dim} \mathfrak{n}_{-1}=$ $2 n\left|m, \operatorname{dim} \mathfrak{n}_{-2}=1\right| 0$, является алгеброй Гейзенберга. Это означает, что компонента $\mathfrak{n}_{-1}$ снабжена невырожденной суперкососимметрической билинейной формой $\omega, \mathrm{a}$ скобка $[\cdot, \cdot]: \mathfrak{n}_{-1} \times \mathfrak{n}_{-1} \rightarrow \mathfrak{n}_{-2}$ задается формулой $[x, y]=\omega(x, y) z$, где $z$ - это фиксированный базисный элемент компоненты $\mathfrak{n}_{-2}$. Полным продолжением алгебры $\mathfrak{n}$ является супералгебра Ли $\mathfrak{k}(2 n+1 \mid m)=\bigoplus_{k \geqslant-2} \mathfrak{k}_{k}$ контактных векторных полей.

В примере 2 мы рассмотрим чисто четный случай ( $m=0, n$ произвольно), а в примере 3 - специальный случай $n=0, m=6$, соответствующий вложению простой исключительной супералгебры Ли $\mathfrak{k} \mathfrak{a} \subset \mathfrak{k}(1 \mid 6)$.

Пример 2. П Пусть $\mathfrak{n}=\mathfrak{n}_{-1} \oplus \mathfrak{n}_{-2}$ есть алгебра Гейзенберга, $\operatorname{dim} \mathfrak{n}_{-1}=2 n$, $\operatorname{dim} \mathfrak{n}_{-2}=1$. Полное продолжение алгебры $\mathfrak{n}-$ это алгебра Ли $\mathfrak{k}(2 n+1)=\bigoplus_{k \geqslant-2} \mathfrak{k}_{k}$ контактных векторных полей. Осуществляя вложение $\mathfrak{n}$ в векторные поля $\mathfrak{v e c t}(2 n+1)=\mathfrak{v e c t}\left(p_{1}, \ldots, p_{n}, q_{1}, \ldots, q_{n}, t\right)$ с градуировкой

$$
\operatorname{deg} t=2, \quad \operatorname{deg} p_{i}=\operatorname{deg} q_{i}=1
$$

для всех $i$, мы можем в качестве $X$-векторов взять, например, поля

$$
X_{q_{i}}=\partial_{q_{i}}+p_{i} \partial_{t}, \quad X_{p_{i}}=\partial_{p_{i}}-q_{i} \partial_{t} ; \quad X_{t}=\partial_{t} .
$$

Тогда $\mathfrak{g}_{-}=\operatorname{Span}\left\{X_{p_{1}}, \ldots, X_{p_{n}}, X_{q_{1}}, \ldots, X_{q_{n}}, X_{t}\right\}$, а контактные векторные поля сохраняют распределение $\mathcal{D}$, выделяемое уравнением Пфаффа $\alpha(X)=0$ на векторное поле $X$, где $\alpha=d t+\sum_{i}\left(p_{i} d q_{i}-q_{i} d p_{i}\right)$. $Y$-векторы имеют вид

$$
Y_{q_{i}}=\partial_{q_{i}}-p_{i} \partial_{t}, \quad Y_{p_{i}}=\partial_{p_{i}}+q_{i} \partial_{t} ; \quad Y_{t}=\partial_{t}
$$

В данном частном случае контактное векторное поле $K$ определяется единственной производящей функцией $F$, которая есть не что иное, как коэффициент при $Y_{t}$ в разложении поля $K$ по базису $Y$, и никаких ограничений на функцию $F$ нет. Вводя обозначаение $F=2 f$ и решая уравнения (18), мы получаем формулу для произвольного контактного векторного поля $K_{f}$ :

$$
\begin{aligned}
K_{f} & =2 f Y_{t}+\sum_{i}\left(Y_{p_{i}}(f) Y_{q_{i}}-Y_{q_{i}}(f) Y_{p_{i}}\right)= \\
& =(2-E)(f) \partial_{t}+\frac{\partial f}{\partial t} E+\sum_{i}\left(\frac{\partial f}{\partial p_{i}} \partial_{q_{i}}-\frac{\partial f}{\partial q_{i}} \partial_{p_{i}}\right),
\end{aligned}
$$

где $E=\sum_{i}\left(p_{i} \partial_{p_{i}}+q_{i} \partial_{q_{i}}\right)$. Это в точности стандартная формула для контактного векторного поля с производящей функцией $f$. В дальнейшем мы используем реализацию алгебры $\mathfrak{k}(2 n+1)$ в производящих функциях $f$.

Если $\mathfrak{h}_{0} \neq \mathfrak{k}_{0}$, то компонента $\mathfrak{h}_{0}$ является определяющей. Компонента $\mathfrak{k}_{0}$ порождается однородными многочленами второй степени в градуировке (35) от переменных $p, q, t$. Следовательно, в качестве базиса $Z$ в $\mathfrak{k}_{0}$ можно взять мономы $p_{i} p_{j}, q_{i} q_{j}, p_{i} q_{j}$ и $t$, а в качестве базиса $Z^{*}$ в двойственном пространстве - операторы

$$
Y_{p_{i}} Y_{p_{j}}, \quad Y_{q_{i}} Y_{q_{j}}, \quad Y_{p_{i}} Y_{q_{j}} \quad \text { при } i \neq j ; \quad \frac{1}{2}\left(Y_{p_{i}} Y_{q_{i}}+Y_{q_{i}} Y_{p_{i}}\right), \quad Y_{t} .
$$


Чтобы описать полное продолжение подалгебры $\mathfrak{g}_{-} \oplus \mathfrak{h}_{0}$, нужно выделить подпространство $\mathfrak{h}_{0}$ в $\mathfrak{k}_{0}$ с помощью условий, сформулированных в терминах линейных уравнений на коэффициенты элементов $\mathfrak{h}_{0}$ в базисе $Z$, а затем переписать эти уравнения в терминах $Z^{*}$.

Если, например, $\mathfrak{h}_{0}=\mathfrak{s p}(2 n)$, то производящие функции не зависят от $t$, и это означает, что $Y_{t}(f)=0$. Это уравнение выделяет в алгебре $\mathfrak{k}(2 n+1)$ пуассонову подалгебру $\mathfrak{p o}(2 n)$.

Если же $\mathfrak{h}_{0}=\mathbb{C} \cdot \mathrm{id}$, то производящие функции $f$ имеют нулевые коэффициенты при всех базисных мономах, кроме $t$, что эквивалентно системе уравнений

$$
\begin{gathered}
Y_{p_{i}} Y_{p_{j}}(f)=0, \quad Y_{q_{i}} Y_{q_{j}}(f)=0, \quad Y_{p_{i}} Y_{q_{j}}(f)=0, \quad i \neq j ; \\
\left(Y_{p_{i}} Y_{q_{i}}+Y_{q_{i}} Y_{p_{i}}\right)(f)=0 .
\end{gathered}
$$

При $i \neq j$ из этих уравнений получаем

$$
Y_{q_{i}} Y_{p_{i}} Y_{p_{j}}(f)-Y_{p_{i}} Y_{q_{i}} Y_{p_{j}}(f)=Y_{t} Y_{p_{j}}(f)=Y_{p_{j}}\left(Y_{t}(f)\right)=0 .
$$

Аналогично можно показать, что $Y_{q_{j}}\left(Y_{t}(f)\right)=0$, а тогда $Y_{t}\left(Y_{t}(f)\right)=0$, и поэтому все частные производные функции $Y_{t}(f)$ равны нулю, а функция $Y_{t}(f)$ постоянна. Тогда $f=c t+f_{0}(p, q)$, причем $\operatorname{deg}_{p, q} f_{0} \leqslant 1$ в силу (37). Стало быть, все продолжение алгебры $\mathfrak{g}_{-} \oplus \mathfrak{g}_{0}$ тривиально (совпадает с ней самой).

Пусть теперь $\mathfrak{h}_{0}=\mathfrak{k}_{0}$ и $\mathfrak{h}_{1} \subset \mathfrak{k}_{1}$. Будучи $\mathfrak{k}_{0}$-модулем, компонента $\mathfrak{k}_{1}$ разлагается в прямую сумму двух $\mathfrak{g}_{0}$-инвариантных подпространств над $\mathbb{C}$ или $\mathbb{R}$ (для char $\mathbb{K}=3$, а в суперслучае даже над $\mathbb{C}$ и $\mathbb{R}$, ситуация более сложная): подпространства $W_{1}$, натянутого на мономы степени три от $p_{i}$ и $q_{j}$, и подпространства $W_{2}$, натянутого на мономы вида $t p_{i}$ и $t q_{j}$. Двойственный базис составляют соответственно симметрические многочлены третьей степени от операторов $Y_{p_{i}}$ и $Y_{q_{j}}$ и операторы $Y_{t} Y_{p_{i}}$, $Y_{t} Y_{q_{j}}$.

Таким образом, подпространство $W_{1}$ определяется условиями

$$
Y_{p_{j}} Y_{t}(f)=Y_{q_{j}} Y_{t}(f)=0 \Longrightarrow Y_{t}(f)=\mathrm{const},
$$

которые выделяют из контактной алгебры $\mathfrak{k}(2 n+1)$ алгебру дифференцирований пуассоновой алгебры $\mathfrak{d e r}(\mathfrak{p o}(2 n))=\mathfrak{p o}(2 n) \oplus \mathbb{C} K_{t}$.

Подпространство $W_{2}$ определяется системой уравнений

$$
\begin{gathered}
Y_{p_{i}} Y_{p_{j}} Y_{p_{k}}(f)=0, \quad Y_{q_{i}} Y_{q_{j}} Y_{q_{k}}(f)=0, \\
Y_{p_{i}} Y_{p_{j}} Y_{q_{k}}(f)=0, \quad Y_{p_{k}} Y_{q_{i}} Y_{q_{j}}(f)=0, \quad k \neq i, j ; \\
Y_{p_{i}}\left(Y_{p_{j}} Y_{q_{j}}+Y_{q_{j}} Y_{p_{j}}\right)(f)=0, \quad Y_{q_{i}}\left(Y_{p_{j}} Y_{q_{j}}+Y_{q_{j}} Y_{p_{j}}\right)(f)=0, \quad i \neq j ; \\
\left(Y_{p_{i}}^{2} Y_{q_{i}}+Y_{p_{i}} Y_{q_{i}} Y_{p_{i}}+Y_{q_{i}} Y_{p_{i}}^{2}\right)(f)=0, \\
\left(Y_{q_{i}}^{2} Y_{p_{i}}+Y_{q_{i}} Y_{p_{i}} Y_{q_{i}}+Y_{p_{i}} Y_{q_{i}}^{2}\right)(f)=0,
\end{gathered}
$$

из которой следует, что функция $Y_{t}(f)$ удовлетворяет уравнениям $(37)$, и, стало быть,

$$
\frac{\partial f}{\partial t} \in \operatorname{Span}\left\{1, p_{1}, \ldots, p_{n}, q_{1}, \ldots, q_{n}, t\right\}
$$


тогда как

$$
f \in \operatorname{Span}\left\{1, p_{1}, \ldots, p_{n}, q_{1}, \ldots, q_{n}, t, t p_{1}, \ldots, t p_{n}, t q_{1}, \ldots, t q_{n}, t^{2}\right\} .
$$

Следовательно, частичное продолжение $\left(\mathfrak{k}_{-} \oplus \mathfrak{k}_{0} \oplus W_{2}\right)_{*}$ изоморфно $\mathfrak{s p}(2 n+2)$.

ПримеР 3. Пусть $\mathfrak{n}=\mathfrak{n}_{-1} \oplus \mathfrak{n}_{-2}-$ супералгебра Гейзенберга $\mathfrak{h e i}(1 \mid 6), \operatorname{dim} \mathfrak{n}_{-1}=$ $0\left|6, \quad \operatorname{dim}_{\mathfrak{n}_{-2}}=1\right| 0$. Полным продолжением алгебры $\mathfrak{n}$ является контактная супералгебра $\mathfrak{g}=\mathfrak{k}(1 \mid 6)$ с нулевой компонентой $\mathfrak{g}_{0}=\mathfrak{c o}(6)$. Компонента $\mathfrak{g}_{1}$ при этом состоит из трех неприводимых $\mathfrak{g}_{0}$-модулей.

Если мы рассмотрим супералгебру $\mathfrak{k}(1 \mid 6)$, реализованную производящими функциями от $t, \theta_{1}, \ldots, \theta_{6}$, т.е. случай

$$
K_{f}=(2-E)(f) \partial_{t}+\frac{\partial f}{\partial t} E-(-1)^{p(f)} \sum_{i} \frac{\partial f}{\partial \theta_{i}} \partial_{\theta_{i}}, \quad f \in \mathbb{C}\left[t, \theta_{1}, \ldots, \theta_{6}\right],
$$

где $E=\sum_{i} \theta_{i} \partial_{\theta_{i}}$ и $\left\{\theta_{i}, \theta_{j}\right\}_{k . b .}=\delta_{i j}$, то $\mathfrak{g}_{1} \simeq t \Lambda(\theta) \oplus \Lambda^{3}(\theta)=t \Lambda(\theta) \oplus \mathfrak{g}_{1}^{+} \oplus \mathfrak{g}_{1}^{-}$, где подмодули $\mathfrak{g}_{1}^{ \pm} \subset \Lambda^{3}(\theta)$ выделяются с помощью звездочки Ходжа:

$$
\mathfrak{g}_{1}^{ \pm}=\left\{f \in \Lambda^{3}(\theta) \mid f^{*}= \pm \sqrt{-1} f\right\} .
$$

Напомним, что двойственность Ходжа * есть не что иное, как преобразование Фурье от нечетных переменных, причем $t$ рассматривается как параметр:

$$
*: f(\xi, t) \mapsto f^{*}(\eta, t)=\int e^{\sum \eta_{i} \xi_{i}} f(\xi, t) \operatorname{vol}(\xi) .
$$

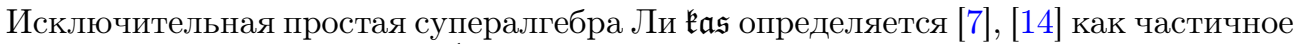
продолжение алгебры $\mathfrak{h}=\bigoplus_{k=-2}^{1} \mathfrak{h}_{k}$, где $\mathfrak{h}_{k}=\mathfrak{k}(1 \mid 6)_{k}$ при $-2 \leqslant k \leqslant 0$ и $\mathfrak{h}_{1}=$ $t \Lambda(\theta) \oplus \mathfrak{g}_{1}^{+}$. Следовательно, $\mathfrak{h}_{1}$ является определяющей компонентой.

При этом

$$
\begin{array}{rlrl}
X_{i}=\partial_{\theta_{i}}+\theta_{i} \partial_{t}, & i=1, \ldots, 6 ; & X_{7}=\partial_{t} ; \\
Y_{i}=\partial_{\theta_{i}}-\theta_{i} \partial_{t}, \quad i=1, \ldots, 6 ; & Y_{7}=X_{7} .
\end{array}
$$

Пусть $I=\left\{i_{1}, i_{2}, i_{3}\right\} \subset\{1, \ldots, 6\}$ - упорядоченный набор индексов, $I^{*}=\left\{j_{1}, j_{2}, j_{3}\right\}$ - двойственный ему набор индексов (другими словами, $\left\{I, I^{*}\right\}$ - четная перестановка индексов $\{1, \ldots, 6\})$. Положим $Y_{I}=Y_{i_{1}} Y_{i_{2}} Y_{i_{3}}, Y_{I^{*}}=Y_{j_{1}} Y_{j_{2}} Y_{j_{3}}$ и определим $\Delta_{Y_{I}}: \mathbb{C}[t, \theta] \rightarrow \mathbb{C}[t, \theta]$ уравнениями

$$
\Delta_{Y_{I}}(f)=(-1)^{p(f)} Y_{I}(f) .
$$

Заметим, что $\Delta_{Y_{I}}\left(t \theta_{s}\right)=0$ для любых $s=1, \ldots, 6$. Следовательно, $\mathfrak{h}_{1}$ можно выделить из компоненты $\mathfrak{k}(1 \mid 6)_{1}$ следующими десятью уравнениями, параметризованными разбиениями $\left(I, I^{*}\right)$ множества $\{1, \ldots, 6\}$, образующими четную подстановку:

$$
\left(\Delta_{Y_{I}}-\sqrt{-1} \Delta_{Y_{I}^{*}}\right)(f)=0 .
$$

Ясно, что эта система эквивалентна системе $\left(Y_{I}-\sqrt{-1} Y_{I^{*}}\right)(f)=0$. Решения последней системы образуют подпространство в пространстве производящих функций, натянутое на следующие функции:

$$
\begin{gathered}
f(t)-\sqrt{-1} f^{\prime \prime \prime}(t) 1^{*}, \quad f_{j}(t) \theta_{j}-\sqrt{-1} f_{j}^{\prime \prime}(t) \theta_{j}^{*}, \\
f_{j k}(t) \theta_{j} \theta_{k}-\sqrt{-1} f_{j k}^{\prime}(t)\left(\theta_{j} \theta_{k}\right)^{*}, \quad f_{j k l}(t)\left(\theta_{j} \theta_{k} \theta_{l}-\sqrt{-1}\left(\theta_{j} \theta_{k} \theta_{l}\right)^{*}\right) .
\end{gathered}
$$


В формулах (41) $j, k, l$ - различные индексы от 1 до 6 . Это описание согласуется с формулами из работы [14].

Благодарности. Работа выполнена при частичной поддержке РФФИ (грант № 05-01-00001). Автор выражает глубокую благодарность Д. А. Лейтесу за стимулирование написания данной работы и чрезвычайно полезные замечания.

\section{Список литературы}

[1] É. Cartan, Leipziger Berichte, XLV (1893), 395-420; "Über die Einfachen Transformationsgrouppen.", Euvres complètes. Partie I. Groups de Lie (Reprinted, 2nd ed.), Éditions du Centre National de la Recherche Scientifique (CNRS), Paris, 1984, 107-132.

[2] P. Grozman, D. Leites, Lett. Math. Phys., 74:3 (2005), 229-262; math.RT/0509400.

[3] P. Grozman, SuperLie, http://www.equaonline.com/math/SuperLie.

[4] Q.-Y. Fei, G.-Y. Shen, J. Algebra, 152 (1992), 439-453.

[5] K. Yamaguchi, "Differential systems associated with simple graded Lie algebras", Progress in Differential Geometry, Adv. Stud. Pure Math., 22, ed. K. Shiohama, Kinokunia, Tokyo, 1993, 413-494.

[6] И. М. Щепочкина, Функи. анализ и его прилож., 33:3 (1999), 59-72; I. Shchepochkina, G. Post, Internat. J. Algebra Comput., 8:4 (1998), 479-495.

[7] I. Shchepochkina, Represent. Theory, 3 (1999), 373-415; hep-th 9702121.

[8] T. Larsson, Structures preserved by consistently graded Lie superalgebras, math-ph/0106004.

[9] T. Larsson, Structures preserved by exceptional Lie algebras, math-ph/0301006.

[10] Ч. Бурдик, П. Грозман, Д. Лейтес, А. Сергеев, ТМФ, 142:2 (2000), 227-238.

[11] V. Molotkov, Explicit realization of induced and coinduced modules over Lie superalgebras by differential operators, math.RT/0509105.

[12] Б. А. Дубровин, С.П. Новиков, А.Т. Фоменко, Современная геометрия. Методы и приложения, Наука, М., 1979.

[13] С. Стернберг, Лекиии по дифберенииалъной геометрии, Мир, М., 1970.

[14] S.-J. Cheng, V. G. Kac, Commun. Math. Phys., 186:1 (1997), 219-231.

Поступила в редакцию 21.IX.2005 г., после доработки 8.XII.2005 г. 